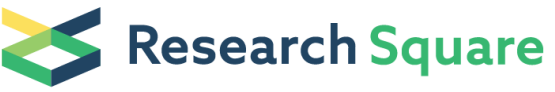

\section{Resonant tunneling driven metal-insulator transition in double quantum-well structures of strongly correlated oxide}

\section{Ryu Yukawa}

High Energy Accelerator Research Organization (KEK) https://orcid.org/0000-0002-5066-9445

\section{Masaki Kobayashi}

University of Tokyo

Daisuke Shiga

Tohoku University

\section{Kohei Yoshimatsu}

Tokyo Institute of Technology

\section{Shoji Ishibashi}

National Institute of Advanced Industrial Science and Technology

\section{Makoto Minohara}

High Energy Accelerator Research Organization (KEK)

\section{Miho Kitamura}

High Energy Accelerator Research Organization (KEK)

\section{Koji Horiba}

Condensed Matter Research Center and Photon Factory, Institute of Materials Structure Science, High Energy Accelerator Research Organization (KEK)

\section{Andres Santander-Syro}

University of Paris-Saclay https://orcid.org/0000-0003-3966-2485

Hiroshi Kumigashira ( $\square$ kumigashira@tohoku.ac.jp )

Tohoku University https://orcid.org/0000-0003-4668-2695

\section{Article}

Keywords: Correlated Materials, Semiconductor Transistors, Wave-function Engineering, Mott-transistors, Angle-resolved Photoemission Spectroscopy,

Posted Date: February 24th, 2021

DOI: https://doi.org/10.21203/rs.3.rs-153757/v1 
License: (c) (i) This work is licensed under a Creative Commons Attribution 4.0 International License. Read Full License

Version of Record: A version of this preprint was published at Nature Communications on December 1st, 2021. See the published version at https://doi.org/10.1038/s41467-021-27327-z. 


\section{Resonant tunneling driven metal-insulator transition in}

4 double quantum-well structures of strongly correlated oxide

${ }^{1}$ Photon Factory, Institute of Materials Structure Science, High Energy Accelerator Research

10 Organization (KEK), Tsukuba, 305-0801, Japan

$11{ }^{2}$ Institute of Multidisciplinary Research for Advanced Materials (IMRAM), Tohoku University, Sendai, 980-8577, Japan

$13{ }^{3}$ Research Center for Computational Design of Advanced Functional Materials, National 14 Institute of Advanced Industrial Science and Technology (AIST), Tsukuba, Ibaraki 305-8568, 15 Japan

$16{ }^{4}$ Université Paris-Saclay, CNRS, Institut des Sciences Moléculaires d'Orsay, 91405, Orsay, 17 France

$19 \dagger$ These authors contributed equally to this work.

20 *e-mail: kumigashira@tohoku.ac.jp

\section{Abstract}

23 The metal-insulator transition (MIT), a fascinating phenomenon occurring in some strongly 24 correlated materials, is of central interest in modern condensed-matter physics. 25 Controlling the MIT by external stimuli is a key technological goal for applications in future 26 electronic devices. However, the standard control by means of the field effect, which works 27 extremely well for semiconductor transistors, faces severe difficulties when applied to the 
MIT. Hence, a radically different approach is needed. Here, we report an MIT induced by resonant tunneling $(\mathrm{RT})$ in double quantum well $(\mathrm{QW})$ structures of strongly correlated oxides. In our structures, two layers of the strongly correlated conductive oxide $\mathrm{SrVO}_{3}$ (SVO) sandwich a barrier layer of the band insulator $\mathrm{SrTiO}_{3}$ (STO). The top QW is a marginal Mott-insulating SVO layer, while the bottom QW is a metallic SVO layer. Angleresolved photoemission spectroscopy experiments reveal that the top QW layer becomes metallized when the thickness of the tunneling barrier layer is reduced. An analysis based on band structure calculations indicates that RT between the quantized states of the double QW induces the MIT. Our work opens avenues for realizing the Mott-transistor based on the wave-function engineering of strongly correlated electrons.

Controlling the quantum ground state of a system is essential for applications. The best-known example is semiconductor technology, where the state (conductive or not conductive) of a semiconductor is driven by the so-called field-effect transistor (FET). In the FET, the number of electric charge carriers is controlled by an external voltage ${ }^{1}$.

Some strongly correlated electron materials naturally show a metal-to-insulator transition $(\mathrm{MIT})^{2,3}$. It would be thus highly desirable to control such a MIT in the same way as it is done for semiconductors. However, despite intensive effort, the FET control of the MIT in strongly correlated materials has been so far unsuccessful ${ }^{4-8}$. In fact, such approach faces fundamental difficulties. One is the insufficient carrier density that can be induced by the electric field to cause a filling-controlled MIT (Mott transistor operation) ${ }^{4,5}$. The other is the shortness of the Thomas-Fermi screening length due to $10^{22}-10^{23} \mathrm{~cm}^{-3}$ mobile carriers, which limits the conductive area where MIT occurs 9 . Thus, realistic future applications of the Mott transistor call for a different principle of controlling the $\mathrm{MIT}^{10}$.

54 Now, according to the Mott-Hubbard theory ${ }^{11}$, the ground state in strongly-correlated materials is 55 described by the ratio of the Coulomb interaction $(U)$ to the bandwidth $(W)^{2,3,11}$. When $U<W$, 56 the material is metallic, but it becomes insulating when $U>W$. Therefore, tuning the $U / W$ ratio 57 by some external perturbation would control the MIT, and the practical realization of this idea has 58 been one of the central goals in modern condensed matter physics ${ }^{4-8}$.

60 Here we propose a new approach for the tuning of the $U / W$ ratio, hence the control of the MIT, 
61 using the resonant-tunneling (RT) effects ${ }^{1}$ in double quantum well (QW) structures of strongly 62 correlated oxides. The concept is schematically illustrated in Fig. 1(a). The double QW 63 structure consists of two strongly correlated oxide layers and a barrier layer (insulator). The top 64 QW is a "marginal" Mott insulator, i.e., a material in the insulating Mott phase but in close 65 proximity ( $U$ slightly larger than $W ; U \gtrsim W$ ) to the metallic one, while the bottom QW is a 66 correlated metal. In the marginal Mott-insulating QW, the quantized electron states are localized 67 due to $U \gtrsim W$, leading to a Mott insulating state, but the QW exhibits a transition to a metallic 68 state by applying a small external stimulus. If the RT occurs between the marginal Mott69 insulating QW and metallic QW states, the QW states that are energetically close to each other 70 are hybridized. In this situation, the resultant envelope wave functions extend over the whole 71 double QW structure and consequently electrons in the top QW states may achieve additional 72 spatial freedom through the RT effects, leading to the reduction in effective Coulomb potential. 73 As a result, the marginal Mott insulator is expected to be metallized owing to $U$ becoming smaller 74 than $W$. Since the MIT is caused by the quantum tunneling phenomena, the MIT control avoids 75 the fundamental problems of the FET approach ${ }^{4-10}$, and is also advantageous over conventional filling (bandwidth) control by chemical doping (pressure) in the bulk ${ }^{2}$.

78 To demonstrate the MIT driven by the RT effects, we fabricate double QW structures where layers 79 of the strongly-correlated conductive oxide $\mathrm{SrVO}_{3}(\mathrm{SVO})$ sandwich a barrier layer of $\mathrm{SrTiO}_{3}$ 80 (STO), a band insulator. Being a highly correlated Fermi-liquid (FL) metal with simple $3 d\left(t_{2 g}\right)^{1}$ configuration $^{12-14}$, SVO in ultrathin film grown onto STO is known to form QW states ${ }^{15,16}$, and to undergo a thickness-dependent transition from the FL metal to a Mott insulator at a critical film 83 thickness of 2-3 monolayers (ML) ${ }^{17,18}$. A recent theoretical study has predicted that the $2 \mathrm{ML}$ 84 of SVO is at the verge of the Mott insulator, and it can easily become a metal by applying a small 85 perturbation $^{19}$. Thus, as the top marginal Mott-insulating QW, we used a 2-ML SVO layer. As 86 a counterpart, for the bottom metallic QW layer we used a 6-ML SVO, so as to induce the RT 87 effect between two energetically close QW states (Supplementary Note 5). Thereafter, the (2$88 \mathrm{ML} \mathrm{SVO}) /(L-\mathrm{ML} \mathrm{STO}) /(6-\mathrm{ML} \mathrm{SVO})$ double QW structure is denoted as $\mathrm{V}_{2} \mathrm{~T}_{L} \mathrm{~V}_{6}$, where $L$ is the 89 thickness of the STO barrier layer. Thus, based on the previously reported structure plot of 90 quantization energies as a function of SVO layer thickness ${ }^{15-17,20,21}$, the first quantization level of 91 the top (2-ML SVO) QW states matches the second quantization level of the bottom (6-ML SVO) 92 QW. Furthermore, in the $\mathrm{V}_{2} \mathrm{~T}_{L} \mathrm{~V}_{6}$ structure, the transition probability of electrons between the 
top and bottom QWs is also controlled as a function of the STO barrier layer thickness $L$, as

94 schematically presented in Fig. 1.

96 The transition from the marginal Mott-insulating QW states to the metallic QW states induced by 97 the RT effects is visualized by in situ angle-resolved photoemission spectroscopy (ARPES). 98 Figure 2a presents a series of ARPES images of the $\mathrm{V}_{2} \mathrm{~T}_{L} \mathrm{~V}_{6}$ double QW structures with varying 99 the STO barrier layer thickness $(L=2,4,10$, and $\infty \mathrm{ML})$. Because these band dispersions have 100 been taken along the $\Gamma$-X direction, the ARPES images consist of only the $d_{z x}$ bands of V $3 d t_{2 g}$ 101 states in the present experimental geometry (see also Supplementary Note 2$)^{15-17,22,23}$. Here, the 102 series of ARPES images are normalized to the incident photon flux; hence, the color scale reflects 103 the change in spectral weight as a function of $L$. Thus, the metallization of top QW will be 104 evidenced by the appearance of a parabolic band at the $\Gamma$ point near the Fermi level $\left(E_{\mathrm{F}}\right)$.

105

106 As can be seen in the ARPES images of the $\mathrm{V}_{2} \mathrm{~T}_{\infty} \mathrm{V}_{6}$ structure, there are no discernible states near $107 E_{\mathrm{F}}$, reflecting the Mott-insulating nature of the 2-ML SVO films. The Mott-insulating state of 108 the top 2-ML QW of $\mathrm{V}_{2} \mathrm{~T}_{\infty} \mathrm{V}_{6}$ is further confirmed by the appearance of the lower Hubbard band 109 at a binding energy of $1.5 \mathrm{eV}$ (see Supplementary Fig. S9) ${ }^{17,18}$. In the $\mathrm{V}_{2} \mathrm{~T}_{4} \mathrm{~V}_{6}$ double $\mathrm{QW}$, as the 110 STO barrier layer becomes thinner (the transition probability of electrons between the two QW 111 states increases), a faint dispersive feature emerges near $E_{\mathrm{F}}$. Eventually, a metallic band whose 112 dispersion crosses $E_{\mathrm{F}}$ is clearly visible in the $\mathrm{V}_{2} \mathrm{~T}_{2} \mathrm{~V}_{6}$ double $\mathrm{QW}$ structures, demonstrating the 113 metallization of the top 2-ML SVO layer. It should be noted that the observed metallic states 114 are at the top 2-ML SVO layer, since the mean-free-path of the photoelectrons in the present 115 experimental condition is about $0.4-0.6 \mathrm{~nm}$ (corresponding to 1.0-1.5 ML in the present case)

116 (refs 24-26). Thus, signals from the buried 6-ML SVO layer are negligible in the ARPES results 117 on $\mathrm{V}_{2} \mathrm{~T}_{L} \mathrm{~V}_{6}$.

\section{8}

119 The emergence of metallic states created by the proximity of two QWs is more clearly seen in the 120 energy distribution curves (EDCs) shown in Figs. 2b, where one observes a systematic evolution 121 of the dispersive metallic states and subsequent reduction in the lower Hubbard band (localized 122 states) in the $\mathrm{V}_{2} \mathrm{~T}_{L} \mathrm{~V}_{6}$ double QW structures. In the top 2-ML SVO layer of the $\mathrm{V}_{2} \mathrm{~T}_{\infty} \mathrm{V}_{6}$ and $123 \mathrm{~V}_{2} \mathrm{~T}_{10} \mathrm{~V}_{6}$ double QW structures, no metallic-band-like features are visible near $E_{\mathrm{F}}$ and only non124 dispersive features are observed below a binding energy of $1 \mathrm{eV}$. The existence of the non125 dispersive structure is caused by spectral weight transfer from the coherent bands near $E_{\mathrm{F}}$ to the 
126 lower Hubbard bands ${ }^{17,18,27}$, indicating the localized nature of V $3 d$ electrons in the top 2-ML SVO

127 layer (see Supplementary Note 4). When the barrier layer thickness decreases, a band-like

128 feature appears near $E_{\mathrm{F}}$ for $\mathrm{V}_{2} \mathrm{~T}_{4} \mathrm{~V}_{6}$, while a weak non-dispersive structure is still visible around

$1290.8 \mathrm{eV}$. Eventually, for the thinnest barrier, the band-like feature evolves to a clear dispersive

130 feature crossing $E_{\mathrm{F}}$.

132 The spectral behavior observed in the top QW layer of $\mathrm{V}_{2} \mathrm{~T}_{L} \mathrm{~V}_{6}$ is similar to that previously 133 observed in thickness-dependent Mott transition in SVO QWs ${ }^{17}$, suggesting the existence of a 134 Mott transition from localized states to standard QW subbands in 2-ML SVO with decreasing $L$.

135 Thus, the next crucial issue is whether the MIT originates from the RT effects or not. The 136 condition for the RT to occur is that there must be an energetic match between quantized states at 137 both the top and bottom QWs ${ }^{1}$. As seen in the 2-ML QW shown in Fig. 2, the bottom of the 138 conduction band (subband bottom energy), corresponding to its first quantization energy, is 139 estimated to be $320 \mathrm{meV}$. This value is consistent with the one extrapolated from the structure

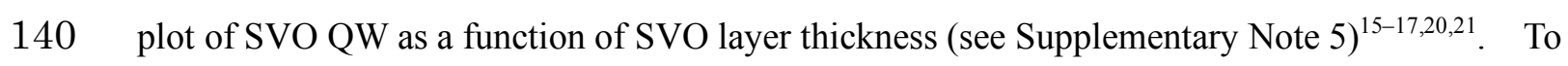

141 confirm the existence of energetically-close QW states for SVO layers at opposite sides of the structure, we investigate the "flip" double QW structure of $\mathrm{V}_{6} \mathrm{~T}_{2} \mathrm{~V}_{2}$ as shown in Fig. 3a, where we

143 only probe the subband structures of the 6-ML QW due to the photoelectron attenuation ${ }^{24-26}$. As

144 expected from previous works ${ }^{15-17}$, the ARPES images consist of the quantized $d_{z x}$ bands with 145 quantum numbers $n=1$ (band bottom at $500 \mathrm{meV}$ ) and $n=2$ (band bottom at $290 \mathrm{meV}$ ). The 146 quantization energy of $n=2$ states for the 6-ML SVO layer is close to that of the metallic band 147 observed for the 2-ML SVO layer $(320 \mathrm{meV})$. The existence of the energetically close QW 148 states in both the top and bottom QW structures suggests the hybridized nature of the envelope 149 wavefunctions of the two subbands ${ }^{28,29}$, leading to the RT effect between the two QWs.

151 The occurrence of the RT effects between the two QW states is further supported by density 152 functional theory (DFT) calculation. Figure 3 compares the DFT results of $\mathrm{V}_{2} \mathrm{~T}_{2} \mathrm{~V}_{6} \mathrm{QW}$ 153 structures with the ARPES results. Owing to the hybridization between the top and bottom QW 154 states, the DFT calculation shows the formation of four $d_{z x}$-derived subbands $\left(n^{\prime}=1-4\right)$ from the 155 bottom. As a result of the hybridization of the original $n=1$ of 2-ML QW and $n=2$ in 6-ML 156 QW, these two energetically close quantization levels form a bonding $\left(n^{\prime}=2\right)$ and an antibonding $157\left(n^{\prime}=3\right)$ state in the double QW. From the DFT calculation, the energy difference between 158 quantization levels $n^{\prime}=2$ and $n^{\prime}=3$ is estimated to be $40 \mathrm{meV}$. In contrast, the $n=1$ and 3 states 
159 in the bottom 6-ML QW are not hybridized to any levels in the top QW and remain unchanged.

160 Judging from the existence probability of electrons belonging to each subband as shown in Fig. $1613 \mathrm{~b}$, the $n^{\prime}=2$ subband, which mainly exists in the 2-ML QW, emerges from the original $n=1$ 162 subband of 2-ML SVO, whereas the other subbands $\left(n^{\prime}=1,3,4\right)$ from the original 6-ML SVO

163 QW states. By considering the escape depth of the present ARPES measurements ${ }^{24-26}$ and the 164 distribution of existence probabilities, only the $n^{\prime}=2$ subband is predominately detectable in 165 ARPES for the $\mathrm{V}_{2} \mathrm{~T}_{2} \mathrm{~V}_{6}$ heterostructure, whereas the $n^{\prime}=1,3,4$ subbands are detectable for the 166 "flip" $\mathrm{V}_{6} \mathrm{~T}_{2} \mathrm{~V}_{2}$ heterostructure. These features are well reproduced in the ARPES results of Figs. $1673 \mathrm{a}$ and $3 \mathrm{c}$, suggesting the occurrence of the RT effects between the top $(n=1)$ and bottom $(n=2)$ 168 QW states.

170 The DFT calculation provides an important indication regarding the metallization of the top QW 171 states: owing to the close proximity of the two quantization energy levels, only $n^{\prime}=2$ and 3 states 172 hybridize with each other. Also, the $n^{\prime}=2\left(n^{\prime}=3\right)$ shows weak but finite existence probabilities 173 in the 6-ML SVO layer (2-ML SVO layer) side, and their respective probability maxima spatially 174 overlap. This spread of the existence probability over both sides of the double QW structure 175 results from the hybridization of the corresponding envelope wavefunctions. Actually, the DFT 176 result demonstrates that about $10 \%$ of the $n^{\prime}=2$ states spread over the 6-ML SVO QW side in the case of $\mathrm{V}_{2} \mathrm{~T}_{2} \mathrm{~V}_{6}$ double QW structures. As schematically illustrated in Fig. 1, in this situation, strongly correlated electrons in the top "marginal” Mott QW states of 2-ML layer SVO $(U \gtrsim W)$

180 the RT effects. The RT effects cause the reduction in effective Coulomb potential $(U<W)$ in 181 the 2-ML QW. As a result, the marginal Mott insulator undergoes an insulator-to-metal 182 transition.

184 The present study demonstrates that the MIT can be controlled by the RT effect in double QW 185 structures of strongly correlated oxides. Our observations offer valuable insight into the quest 186 for novel quantum phenomena using oxide heterostructures ${ }^{6-10,30-33}$, since the $U / W$ ratio can be 187 controlled by designing the wavefunction of their strongly correlated electrons. In addition, 188 from an applied perspective, the MIT control based on the double QW structure studied here has 189 fundamental advantages over conventional FET $\operatorname{control}^{4,5,9}$ : the Mott transition may be operated 190 by aligning two quantization levels through the application of a small voltage, and the entire QW 191 will undergo a MIT irrespective of the limitation imposed by Thomas-Fermi screening. The 
192 present demonstration opens an avenue for creating a Mott-transistor operation based on the

193 quantum RT effects between designed wavefunctions of strongly correlated electrons.

\section{Methods}

197 Laser molecular-beam epitaxy. QW structures were grown on atomically-flat (001) surfaces

198 of $\mathrm{TiO}_{2}$-terminated $\mathrm{Nb}$-doped STO substrates in a laser molecular-beam epitaxy chamber 199 connected to an ARPES system at BL-2A of Photon Factory (PF) $)^{14-18}$. During the growth, the 200 thickness was precisely and digitally controlled on the atomic scale by monitoring the intensity 201 oscillation of reflection high-energy electron diffraction spots. The details of the growth 202 conditions of SVO and STO layers are described elsewhere ${ }^{15-18,34}$. Note that all double QW 203 structures were fabricated under the same conditions as those of the previously reported 204 SVO/STO heterostructures ${ }^{15-18,34}$, wherein atomically-flat surfaces and chemically abrupt 205 interfaces formed. The growth condition details and the characterizations of the QW structures 206 are given in Supplementary Information.

207

208 In situ angle-resolved photoemission spectroscopy. After growth, the samples were 209 transferred to the ARPES chamber under an ultrahigh vacuum of $10^{-10}$ Torr to avoid the 210 degradation of the sample surfaces on exposure to air. The ARPES experiments were conducted 211 in situ at a temperature of $20 \mathrm{~K}$ using horizontal linear polarization of the incident light. The 212 incident photon energy was $88 \mathrm{eV}$. The energy and angular resolutions were respectively set to 213 about $30 \mathrm{meV}$ and $0.3^{\circ}$. The $E_{\mathrm{F}}$ of the samples was calibrated by measuring a gold foil that was 214 electrically connected to the samples. The details of the ARPES measurement setups are given 215 in Supplementary Note 2.

217 Electronic structure calculations. First-principles calculations based on DFT were carried out 218 in the framework of the Perdew-Burke-Ernzerhof-type generalized-gradient approximation ${ }^{35}$ 219 using the computational code of QMAS $^{36}$. The corresponding Brillouin zone was sampled by $2208 \times 8 \times 2 \boldsymbol{k}$-mesh for the self-consistent field calculation. To obtain the electronic density of 221 states, calculations with fixed charges were made at additional $\boldsymbol{k}$ points. We have adopted a 222 repeated slab geometry with vacuum layer in between neighboring slabs of the $V_{2} T_{2} V_{6}$ 223 heterostructure as illustrated in the top panel of Fig. 3b. For the comparison with the ARPES 224 results, the subband dispersion $\left(E_{\text {sub }}\right)$ was obtained by multiplying the calculated band structures 
$225\left(E_{\mathrm{DFT}}\right)$ by the band renormalization factor $(Z): E_{\mathrm{sub}}\left(k_{\|}\right)=Z \cdot E_{\mathrm{DFT}}\left(k_{\|}\right)$, where $k_{\|}$is the 226 momentum parallel to the surface. The value of $Z$ is inversely proportional to the mass 227 enhancement factor, and the mass enhancement in the subbands is known to be associated with 228 strong interaction among V $3 d$ electrons ${ }^{12-17,37-39}$. The best fit to the ARPES results gives $Z=$ 2290.55 (mass enhancement factor $\sim 1.8$ ) for the heterostructure, in good agreement with previous 230 results measured on $\mathrm{SrVO}_{3}$ thin films ${ }^{15}$.

\section{Acknowledgment}

233 The authors are very grateful to Y. Kuramoto, Y. Matsumoto, and A. Fujimori for their useful 234 discussions and acknowledge T. Kanda and E. Sakai for their support in the experiment at PF. 235 This work was financially supported by a Grant-in-Aid for Scientific Research (Nos. 16H02115, 236 16KK0107, 19H01830, and 20KK0117) from the Japan Society for the Promotion of Science 237 (JSPS), CREST (JPMJCR18T1) from the Japan Science and Technology Agency (JST), and the 238 MEXT Element Strategy Initiative to Form Core Research Center (JPMXP0112101001). AFSS 239 is supported by public grants from the Centre National de la Recherche Scientifique (CNRS), 240 project PICS FermiAds No. 272651, and the French National Research Agency (ANR), project 241 Fermi-NESt No. ANR-16-CE92-0018. The work performed at KEK-PF was approved by the 242 Program Advisory Committee (proposals 2018S2-004) at the Institute of Materials Structure 243 Science, KEK.

244

\section{Author contributions}

246 The samples were grown and characterized by R.Y., M. Kobayashi., and M.M. ARPES 247 measurements were performed by R.Y., M. Kobayashi, D.S., M.M., M. Kitamura, and K.H. R.Y. 248 and M. Kobayashi analyzed the ARPES data. R.Y., M. Kobayashi, K.Y., A.F.S-S, and H.K. 249 contributed to the interpretation. S.I. performed the DFT calculation. R.Y., A.F.S-S, and H.K. 250 wrote the paper with input and discussion from all co-authors. H.K. devised the project and 251 were responsible for its overall planning and direction. All authors discussed the results and 252 commented on the manuscript.

\section{Additional information}

255 Supplementary information is available in the online version of the paper. Reprints and 256 permissions information is available online at www.nature.com/reprints. Correspondence and 257 requests for materials should be addressed to H.K. (e-mail: kumigashira@tohoku.ac.jp). 


\section{Competing financial interests}

259 The authors declare no competing financial interests.

260

261

1. Sze, S. M. \& Ng, K. K. Physics of Semiconductor Devices 3rd edn (Wiley-Interscience,

2. Imada, M., Fujimori, A. \& Tokura, Y. Metal-insulator transitions. Rev. Mod. Phys. 70, 1039-1263 (1998).

3. Georges, A., Kotliar, G., Krauth, W. \& Rozenberg, M. J. Dynamical mean-field theory of strongly correlated fermion systems and the limit of infinite dimensions. Rev. Mod. Phys. 68, 13-125 (1996).

4. Ahn, C. H., Triscone, J.-M. \& Mannhart, J. Electric field effect in correlated oxide systems.

5. Ahn, C. H. et al. Electrostatic modification of novel materials. Rev. Mod. Phys. 78, 1185-

6. Takagi, H. \& Hwang, H. Y. An emergent change of phase for electronics. Science 327, 1212 (2006).

7. Mannhart, J. \& Schlom, D. G. Oxide Interfaces--An Opportunity for Electronics. Science 327, 1607-1611 (2010).

8. Hwang, H. Y. et al. Emergent phenomena at oxide interfaces. Nat. Mater. 11, 103-113 (2012).

9. Nakano, M. et al. Collective bulk carrier delocalization driven by electrostatic surface charge accumulation. Nature 487, 459-462 (2012).

10. Tokura, Y., Kawasaki, M. \& Nagaosa, N. Emergent functions of quantum materials. Nat. Phys. 13, 1056-1068 (2017).

11. Hubbard, J. Electron Correlations in Narrow Energy Bands. Proc. R. Soc. A Math. Phys. Eng. Sci. 276, 238-257 (1963).

12. Inoue, I. et al. Systematic Development of the Spectral Function in the $3 d^{1}$ Mott-Hubbard System $\mathrm{Ca}_{1-x} \mathrm{Sr}_{x} \mathrm{VO}_{3}$. Phys. Rev. Lett. 74, 2539-2542 (1995).

13. Inoue, I. H., Goto, O., Makino, H., Hussey, N. E. \& Ishikawa, M. Bandwidth control in a perovskite-type $3 d^{1}$-correlated metal $\mathrm{Ca}_{1-x} \mathrm{Sr}_{x} \mathrm{VO}_{3}$. I. Evolution of the electronic properties and effective mass. Europhys. Lett. 58, 4372-4383 (1998).

291 14. Aizaki, S. et al. Self-Energy on the Low- to High-Energy Electronic Structure of 
15. Yoshimatsu, K. et al. Metallic Quantum Well States in Artificial Structures of Strongly Correlated Oxide. Science 333, 319-322 (2011).

16. Kobayashi, M. et al. Origin of the Anomalous Mass Renormalization in Metallic Quantum Well States of Strongly Correlated Oxide $\mathrm{SrVO}_{3}$. Phys. Rev. Lett. 115, 076801 (2015).

17. Kobayashi, M. et al. Emergence of Quantum Critical Behavior in Metallic Quantum-Well States of Strongly Correlated Oxides. Sci. Rep. 7, 16621 (2017).

18. Yoshimatsu, K. et al. Dimensional-Crossover-Driven Metal-Insulator Transition in $\mathrm{SrVO}_{3}$

301 302

$$
\text { Ultrathin Films. Phys. Rev. Lett. 104, } 147601 \text { (2010). }
$$

19. Zhong, Z. et al. Electronics with Correlated Oxides: $\mathrm{SrVO}_{3} / \mathrm{SrTiO}_{3}$ as a Mott Transistor. Phys. Rev. Lett. 114, 246401 (2015).

20. Okamoto, S. Anomalous mass enhancement in strongly correlated quantum wells. Phys. Rev. B 84, 201305 (2011).

21. Zhong, Z., Zhang, Q. \& Held, K. Quantum confinement in perovskite oxide heterostructures: Tight binding instead of a nearly free electron picture. Phys. Rev. B 88, 125401 (2013).

22. Meevasana, W. et al. Creation and control of a two-dimensional electron liquid at the bare $\mathrm{SrTiO}_{3}$ surface. Nat. Mater. 10, 114-118 (2011).

23. Santander-Syro, A. F. et al. Two-dimensional electron gas with universal subbands at the surface of $\mathrm{SrTiO}_{3}$. Nature 469, 189-193 (2011).

24. Zangwill, A. Physics at Surfaces. (Cambridge University Press, 1988).

25. Tanuma, S., Powell, C. J. \& Penn, D. R. Calculations of electron inelastic mean free paths. IX. Data for 41 elemental solids over the $50 \mathrm{eV}$ to $30 \mathrm{keV}$ range. Surf. Interface Anal. 43, 689-713 (2011).

26. Hüfner, S. Photoelectron Spectroscopy: Principles and Applications $3^{\text {rd }}$ edn (Springer, 2003).

27. King, P. D. C. et al. Atomic-scale control of competing electronic phases in ultrathin $\mathrm{LaNiO}_{3}$. Nat. Nanotechnol. 9, 443-447 (2014).

28. Kawasaki, J. K. et al. Rutile $\mathrm{IrO}_{2} / \mathrm{TiO}_{2}$ superlattices: A hyperconnected analog to the Ruddelsden-Popper structure. Phys. Rev. Mater. 2, 054206 (2018).

29. Choi, W. S., Lee, S. A., You, J. H., Lee, S. \& Lee, H. N. Resonant tunnelling in a quantum oxide superlattice. Nat. Commun. 6, 7424 (2015).

30. Lee, D. et al. Isostructural metal-insulator transition in $\mathrm{VO}_{2}$. Science 362, 1037-1040 (2018). 
326

327

328

329

330

331

332

333

334

335

336

337

338

339

340

341

342

343

344

345

346

347
31. Domínguez, C. et al. Length scales of interfacial coupling between metal and insulator phases in oxides. Nat. Mater. 19, 1182-1187 (2020).

32. Ramesh, R. \& Schlom, D. G. Creating emergent phenomena in oxide superlattices. Nat. Rev. Mater. 4, 257-268 (2019).

33. Matsuno, J. et al. Engineering a Spin-Orbital Magnetic Insulator by Tailoring Superlattices. Phys. Rev. Lett. 114, 247209 (2015).

\section{References for Methods}

34. Yoshimatsu, K. et al. Determination of the surface and interface phase shifts in metallic quantum well structures of perovskite oxides. Phys. Rev. B 88, 115308 (2013).

35. Perdew, J. P., Burke, K. \& Ernzerhof, M. Generalized Gradient Approximation Made Simple. Phys. Rev. Lett. 77, 3865-3868 (1996).

36. Ishibashi, S., Tamura, T., Tanaka, S., Kohyama, M. \& Terakura, K. Ab initio calculations of electric-field-induced stress profiles for diamond/ $c-\mathrm{BN}$ (110) superlattices. Phys. Rev. B 76, 153310 (2007).

37. Okamoto, S. \& Millis, A. J. Spatial inhomogeneity and strong correlation physics: A dynamical mean-field study of a model Mott-insulator-band-insulator heterostructure. Phys. Rev. B 70, 241104(R) (2004).

38. Takizawa, M. et al. Coherent and incoherent $d$ band dispersions in $\mathrm{SrVO}_{3}$. Phys. Rev. B 80, 235104 (2009).

39. Nekrasov, I. A. et al. Momentum-resolved spectral functions of $\mathrm{SrVO}_{3}$ calculated by LDA + DMFT. Phys. Rev. B 73, 155112 (2006). 
a

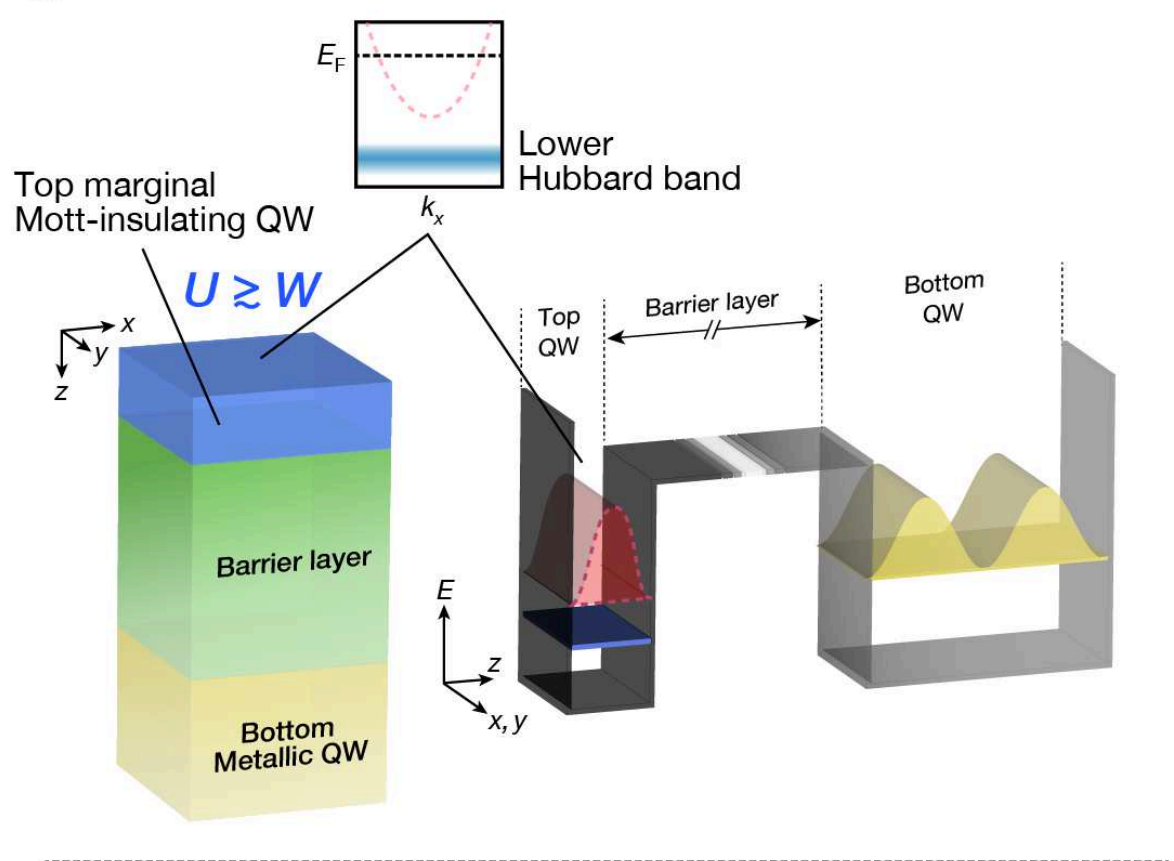

b
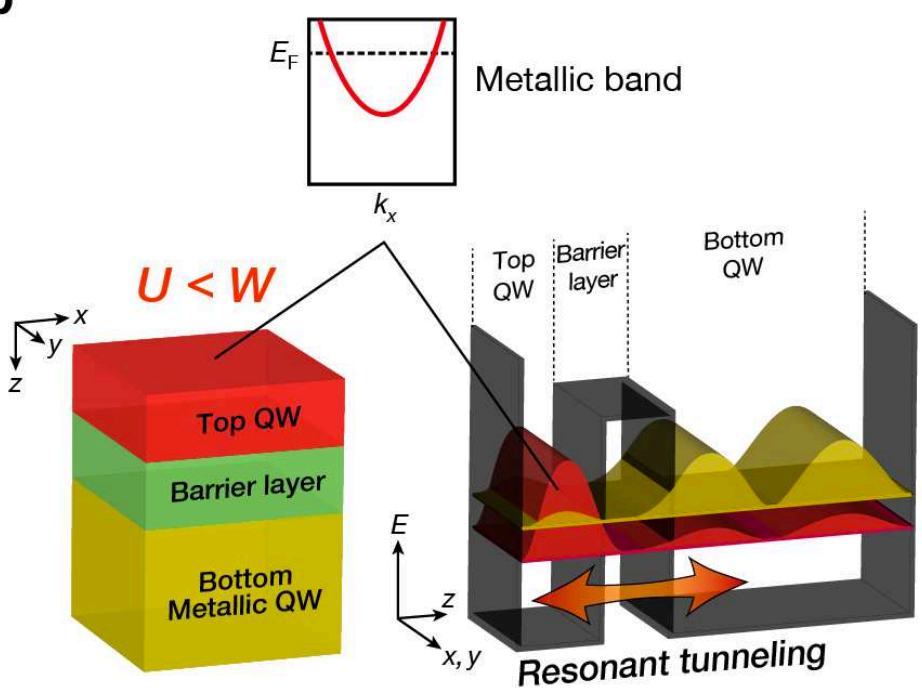

351 Figure 1 | Schematic illustration of metal-insulator transition induced by resonant tunneling

352 effects. a, Before switching on the RT effect. Structure of a double QW composed of a marginal

353 Mott-insulator QW layer/ barrier layer/ metallic QW layer. The marginal Mott-insulator $(U \gtrsim$

$354 W$ ), which undergoes the transition to metal by application of a perturbation, is used as top QW

355 layer. Owing to the long distance between the top and bottom QWs, there is no RT effect

356 between the two QWs. The corresponding band diagram and standing waves are illustrated on 
357 the right side. The potential well is represented as a black plate, while the existence-probability 358 amplitudes of the metallic QW states are drawn in red for the top QW and in yellow for the bottom 359 QW. Owing to the marginal Mott insulating nature of top QW states, the strongly correlated 360 electrons in the original top QW states become localized, resulting in a localized state (lower 361 Hubbard band) presented in blue. b, After switching on the RT effects. Owing to the 362 hybridization between the top and bottom QW states, bonding (red curve) and antibonding 363 (yellow curve) states are formed. In this situation, electrons in the top QW will be able to move 364 to the bottom QW via RT. By gaining the spatial degree of freedom, the effective Coulomb 365 repulsion of electrons is weakened and the top QW undergoes the transition from the marginal 366 Mott insulator $(U \gtrsim W)$ to the metal $(U<W)$. 

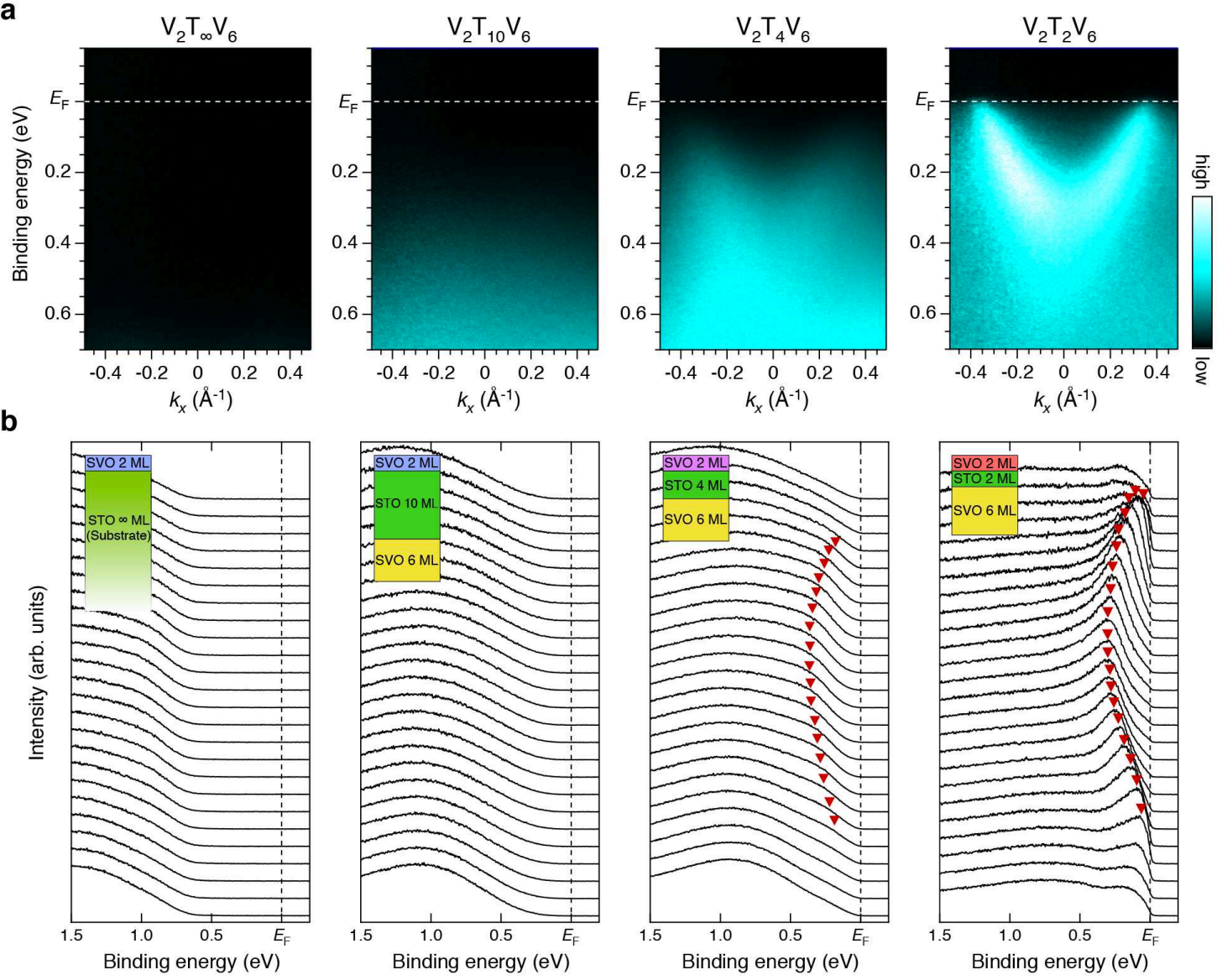

371 Figure 2 | Visualization of the MIT induced by reduction in the barrier-layer thickness. a,

372 Respective ARPES images for $\mathrm{V}_{2} \mathrm{~T}_{L} \mathrm{~V}_{6}$ double $\mathrm{QW}$ structures with $L$ reducing from $\infty$ to 2 . The

373 ARPES data were acquired at a photon energy of $h v=88 \mathrm{eV}$ along a $k_{x}$ slice near the $\Gamma$ point,

374 which includes only the quantized $d_{z x}$ band. Note that the series of ARPES spectra are

375 normalized to the incident photon flux, and the normalized intensity is given by a color scale

376 shown on the right side. b, Corresponding EDCs to the respective ARPES images. The red

377 filled triangles are guides to eye for the dispersive features. The broad non-dispersive feature

378 around $1.0 \mathrm{eV}$ is the lower Hubbard band. The inset shows the schematic side views of the 379 corresponding double QW structures. 
a

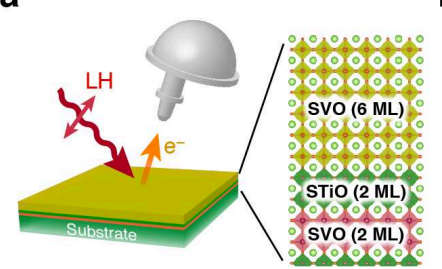

b

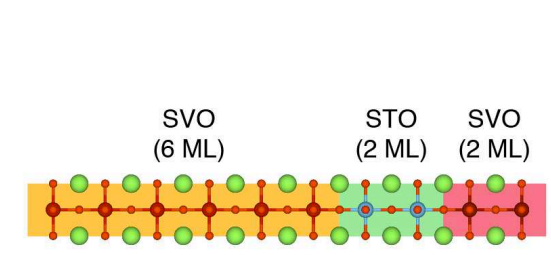

C
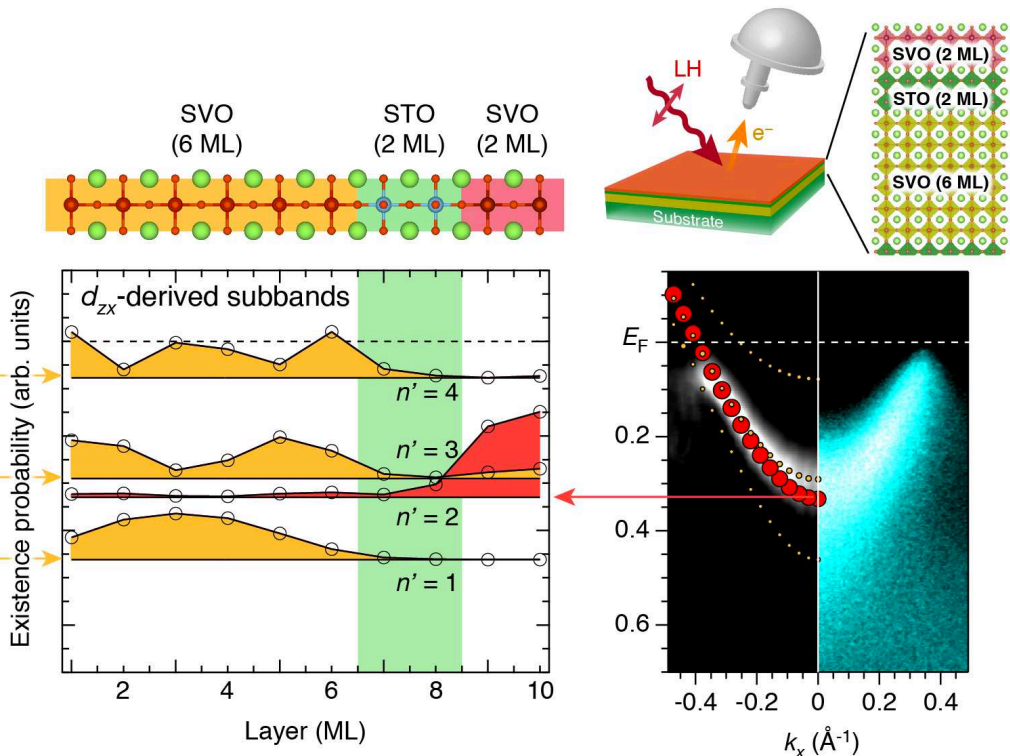
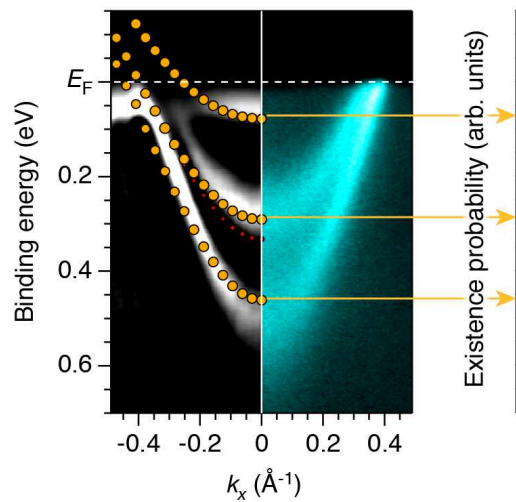

Figure 3 | Subband structures formed at the $V_{2} T_{2} V_{6}$ heterostructure. a, c, Comparison of

ARPES images taken near the $\Gamma$ point with the DFT calculations for $V_{6} T_{2} V_{2}$ (a) and $V_{2} T_{2} V_{6}$ (c) double QWs. Raw ARPES intensity plots are shown on the right half side and peak-enhanced plots (see Supplementary Note 3) on the left side, together with the DFT calculation. As shown in the schematic layout on the top, electrons near the surface $(\sim 0.4-0.6 \mathrm{~nm})$ are detected in the ARPES measurements. Since only $d_{z x}$-derived subbands are detected in this experimental configuration (see Supplementary Note 2), the corresponding subband with predominant $d_{z x}$

391 character (filled circles) is presented in the calculation for comparison (the subbands for all $t_{2 g}$ states are shown in Supplementary Fig. S11). The probability of the electron being detected by ARPES is represented by the size of the filled circles. $\quad \mathbf{b}$, Existence probabilities for respective $d_{z x}$-derived subbands, which are calculated by Mulliken population analysis, are plotted along the $z$ direction for the $\mathrm{V}_{2} \mathrm{~T}_{2} \mathrm{~V}_{6}$ heterostructure. The baselines of the existence probabilities (solid black lines) correspond to the quantization energies (subband minimum energies). The existence probability of bonding $n^{\prime}=2$ states (red hatched) has central weight at the 2-ML QW, while that of antibonding $n^{\prime}=3$ (yellow hatched) at 6-ML QW, representing their original character. 


\section{Figures}

a

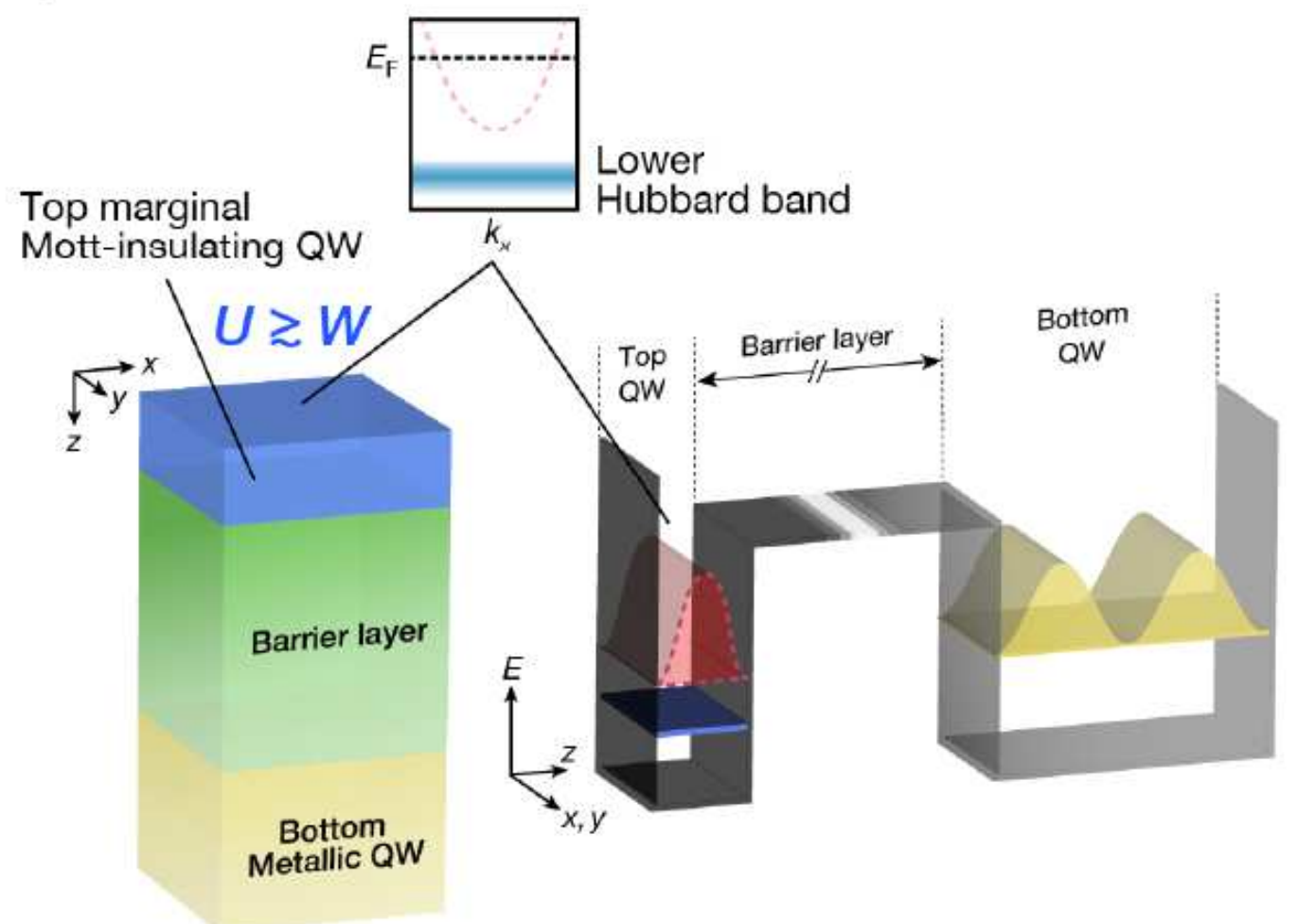

b
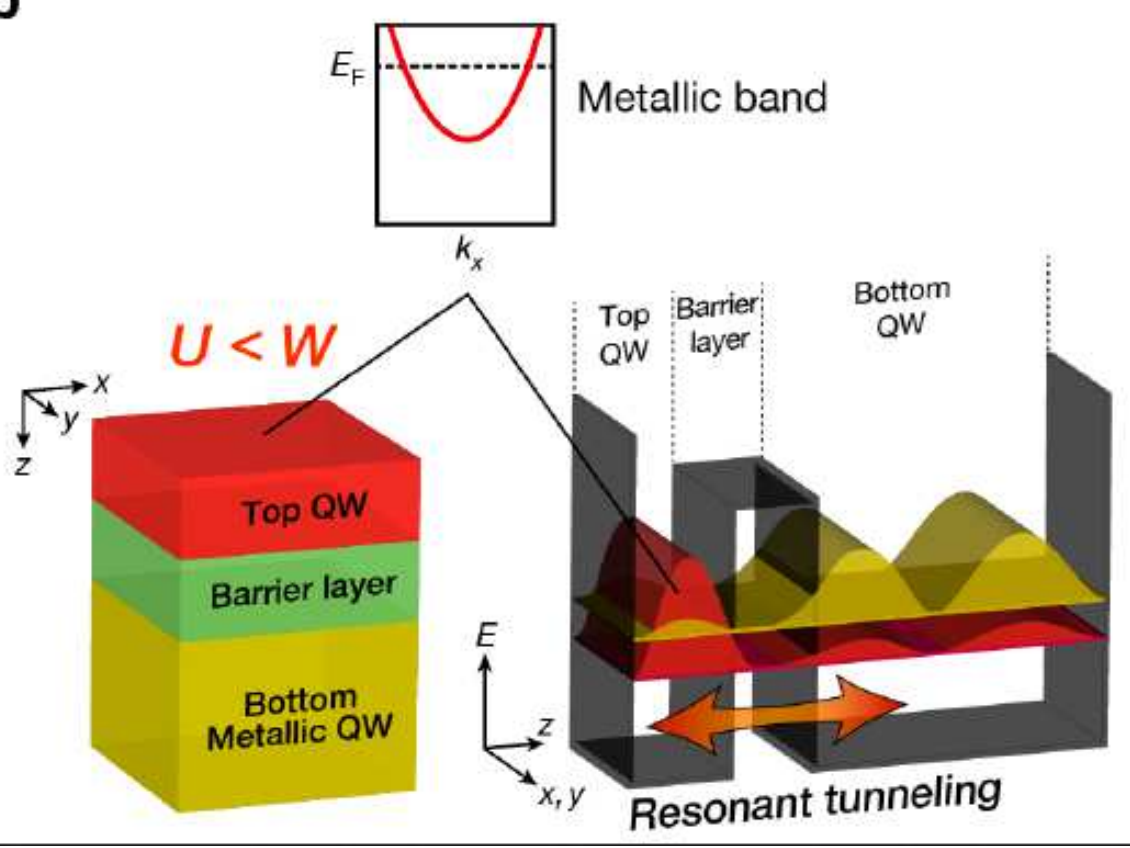

Figure 1

Schematic illustration of metal-insulator transition induced by resonant tunneling effects. a, Before switching on the RT effect. Structure of a double QW composed of a marginal Mott-insulator QW layer/ barrier layer/ metallic $\mathrm{QW}$ layer. The marginal Mott-insulator $(\mathrm{U} \otimes \mathrm{W})$, which undergoes the transition to 
metal by application of a perturbation, is used as top QW layer. Owing to the long distance between the top and bottom QWs, there is no RT effect between the two QWs. The corresponding band diagram and standing waves are illustrated on the right side. The potential well is represented as a black plate, while the existence-probability amplitudes of the metallic QW states are drawn in red for the top QW and in yellow for the bottom QW. Owing to the marginal Mott insulating nature of top QW states, the strongly correlated electrons in the original top QW states become localized, resulting in a localized state (lower Hubbard band) presented in blue. b, After switching on the RT effects. Owing to the hybridization between the top and bottom QW states, bonding (red curve) and antibonding (yellow curve) states are formed. In this situation, electrons in the top QW will be able to move to the bottom QW via RT. By gaining the spatial degree of freedom, the effective Coulomb repulsion of electrons is weakened and the top QW undergoes the transition from the marginal Mott insulator $(U \otimes W)$ to the metal $(U<W)$.

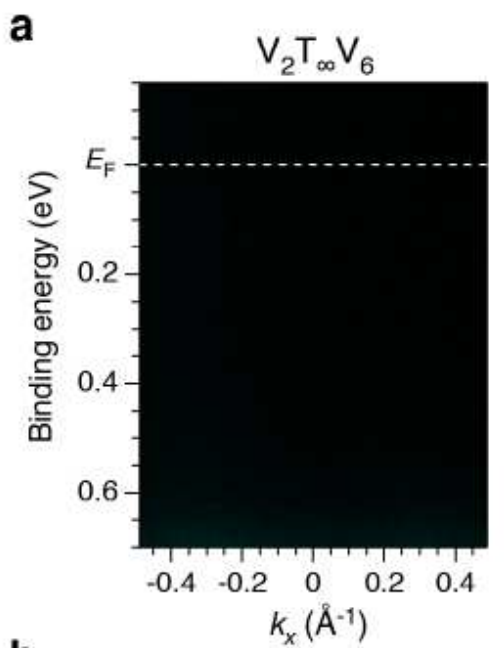

b

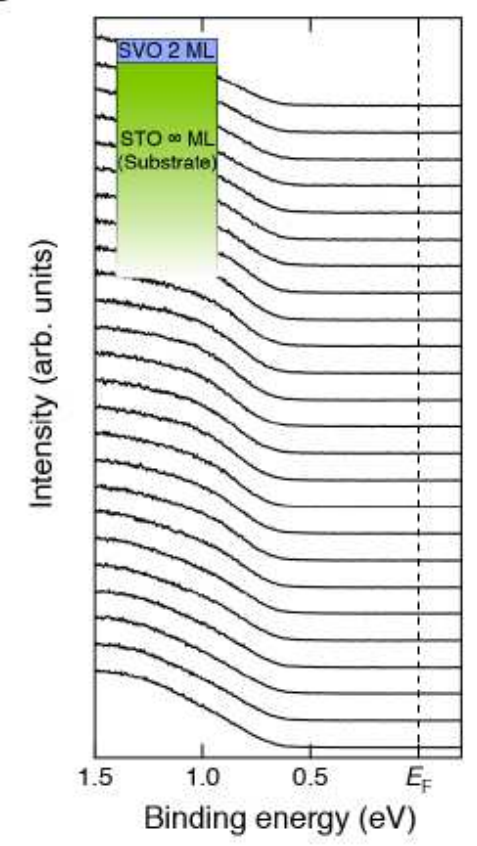

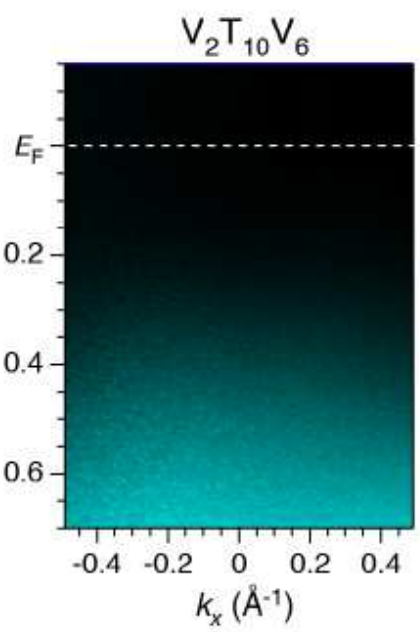

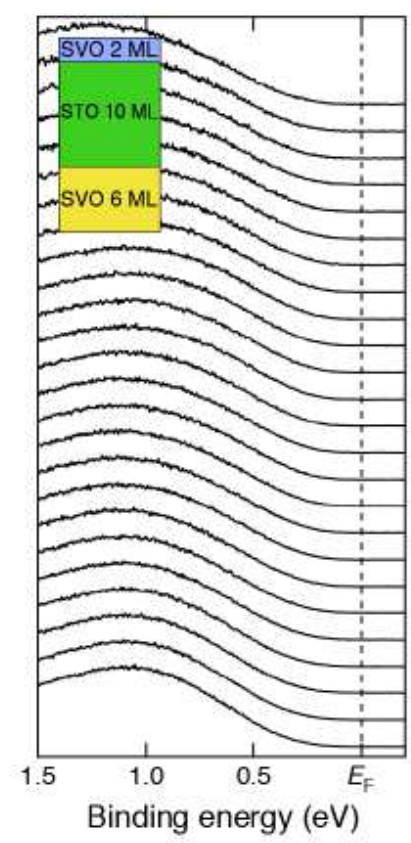

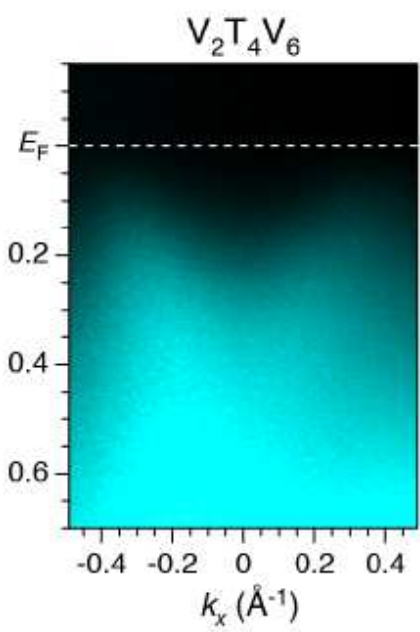

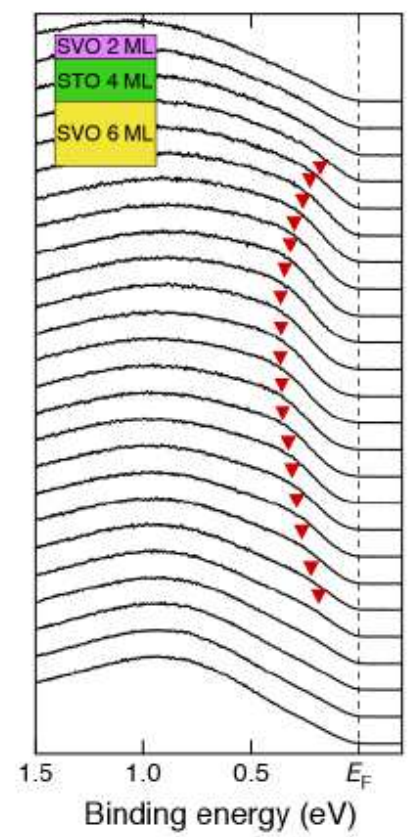

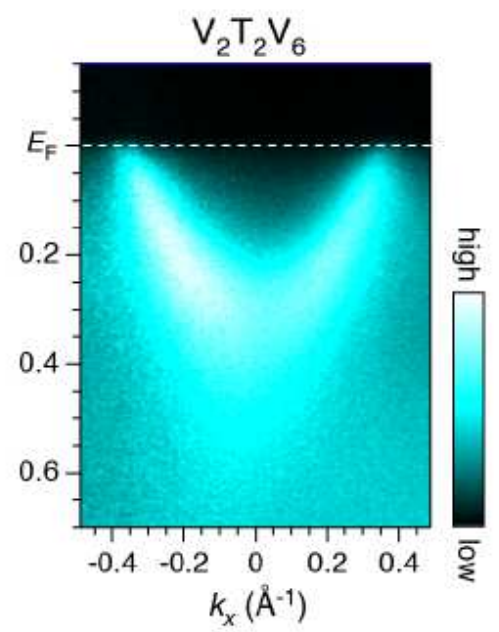

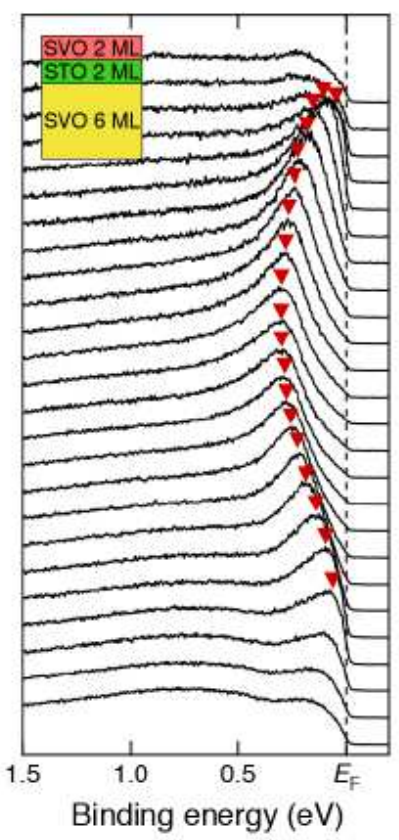

Figure 2 
Visualization of the MIT induced by reduction in the barrier-layer thickness. a, Respective ARPES images for V2TLV6 double QW structures with $L$ reducing from $\infty$ to 2 . The ARPES data were acquired at a photon energy of hv $=88 \mathrm{eV}$ along a kx slice near the $\mathrm{G}$ point, which includes only the quantized dzx band. Note that the series of ARPES spectra are normalized to the incident photon flux, and the normalized intensity is given by a color scale shown on the right side. $b$, Corresponding EDCs to the respective ARPES images. The red filled triangles are guides to eye for the dispersive features. The broad non-dispersive feature around $1.0 \mathrm{eV}$ is the lower Hubbard band. The inset shows the schematic side views of the corresponding double QW structures.

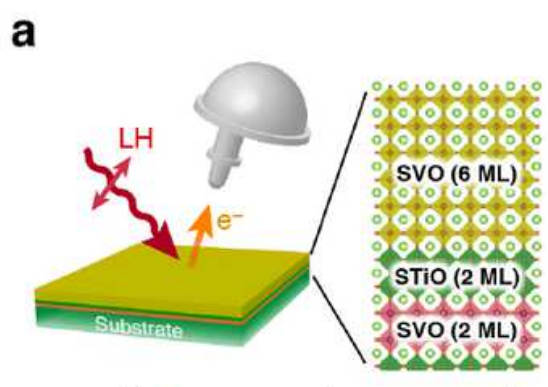

b

C
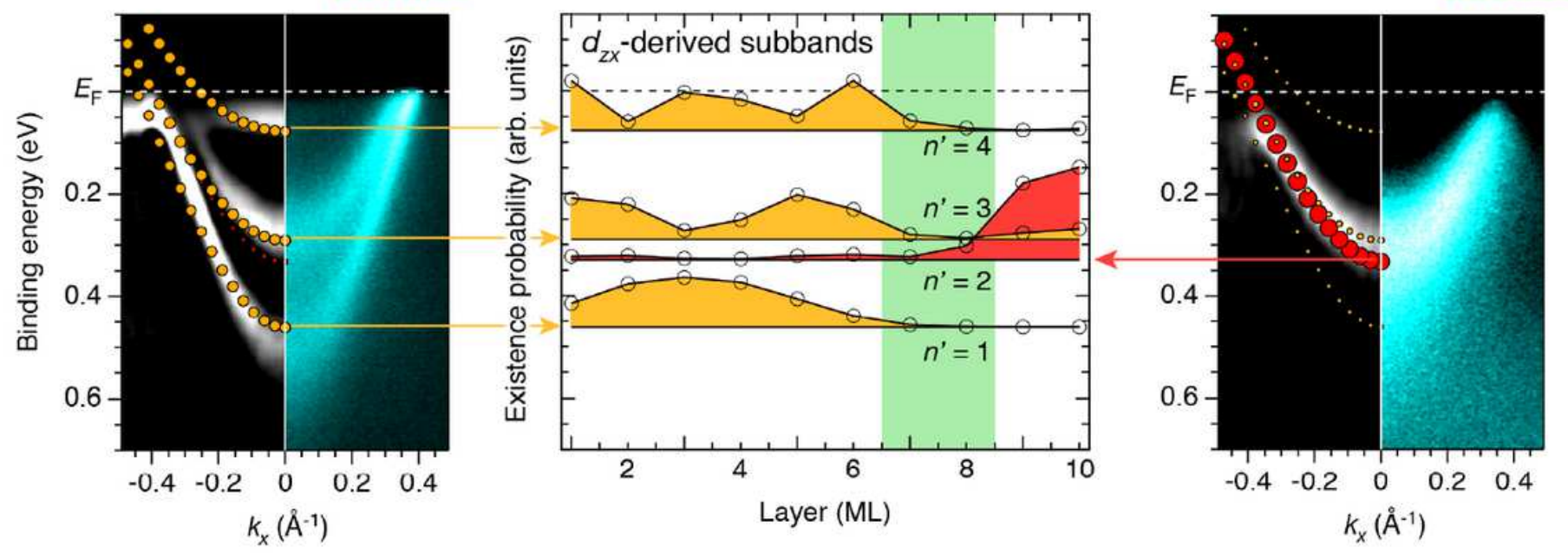

Figure 3

Subband structures formed at the V2T2V6 heterostructure. a, c, Comparison of ARPES images taken near the $\Gamma$ point with the DFT calculations for V6T2V2 (a) and V2T2V6 (c) double QWs. Raw ARPES intensity plots are shown on the right half side and peak-enhanced plots (see Supplementary Note 3 ) on the left side, together with the DFT calculation. As shown in the schematic layout on the top, electrons near the surface $(\sim 0.4-0.6 \mathrm{~nm})$ are detected in the ARPES measurements. Since only dzx-derived subbands are detected in this experimental configuration (see Supplementary Note 2), the corresponding subband with predominant dzx character (filled circles) is presented in the calculation for comparison (the subbands for all t2g states are shown in Supplementary Fig. S11). The probability of the electron being detected by ARPES is represented by the size of the filled circles. b, Existence probabilities for respective dzx-derived subbands, which are calculated by Mulliken population analysis, are plotted along the $z$ direction for the V2T2V6 heterostructure. The baselines of the existence probabilities (solid black lines) correspond to the quantization energies (subband minimum energies). The existence probability of bonding $\mathrm{n}^{\prime}=2$ states 
(red hatched) has central weight at the 2-ML QW, while that of antibonding $\mathrm{n}^{\prime}=3$ (yellow hatched) at 6$\mathrm{ML} Q W$, representing their original character.

\section{Supplementary Files}

This is a list of supplementary files associated with this preprint. Click to download.

- 210123sSVODQWSI.pdf 